Anales de Geografía de la Universidad Complutense ISSN: 0211-9803

http://dx.doi.org/10.5209/ AGUC.53589

\title{
Una década de planes de movilidad urbana sostenible en España 2004- 2014
}

\author{
Pilar Vega Pindado ${ }^{1}$ \\ Recibido: 21 de abril del 2016 / Enviado a evaluar: 22 de mayo del 2016 / Aceptado: 18 de julio del 2016
}

Resumen. Los Planes de Movilidad Urbana Sostenible (PMUS) son una herramienta de reciente implantación en España que en la última década se ha desarrollado en un buen número de municipios. El objetivo es conocer cuál ha sido la dimensión territorial y el impacto que este instrumento de planificación de la movilidad ha tenido en nuestro país entre los años 2004 y 2014. Como resultado se ha logrado describir el panorama de los PMUS en España y su evolución durante esta última década en cada una de las Comunidades Autónomas.

Palabras clave: Plan de Movilidad Urbana Sostenible; Municipio; planificación de movilidad; movilidad integral; movilidad sostenible.

\section{[en] A decade of susteinable urban mobility plans in Spain 2004-2014}

\begin{abstract}
Sustainable Urban Mobility Plan, a tool recently introduced in Spain, have been developed in the last decade in a number of municipalities. The objective of this research is to understand the territorial dimension and the impact this instrument mobility planning has had in our country between 2004 and 2014. As a result, it has been possible to describe the landscape of Sustainable Urban Mobility Plan in Spain and its evolution over the past decade in each of the Autonomous Communities.
\end{abstract}

Key words: Sustainable Urban Movility Plan; municipality; mobility planning; integral mobility; sustainable mobility.

\section{[fr] Une décennie de mobilité urbaine durable prévoit en Espagne 2004-2014}

Résumé. Les plans de mobilité urbaine durable, un outil récemment mis en place en Espagne, ont été développés dans la dernière décennie dans plusieurs municipalités. L'objectif de cette recherche est de comprendre la dimension territoriale et l'impact de cet instrument pour la planification de la mobilité dans notre pays entre 2004 et 2014. En conséquence, il a été possible de décrire la situation des plans en Espagne et son évolution dans la dernière décennie dans chacune des communautés autonomes.

Mots clés: Plans urbains de mobilité intégré et durable; municipalité; planification de la mobilité; mobilité intégrale; mobilité durable.

1 Departamento de Geografía Humana. Universidad Complutense de Madrid.

E-mail: mariapve@pdi.ucm.es 
Cómo citar. Vega Pindado, P. (2016): Una década de planes de movilidad sostenibilidad en España 2004-2014. Anales de Geografía de la Universidad Complutense, 36(2), 351-372.

Sumario. 1. Introducción. 2. Metodología y fuentes. 3. Origen y evolución. 4. Evolución de los PMUS en España (2005-2014). 5. Tercera etapa: Desarrollo y momento (2001-2014). 5.1. Distribución territorial en las Comunidades Autónomas. 5.2. Impacto en el territorio y en la población de los PMUS. 6. Conclusiones. 7. Referencias bibliográficas. 8. Leyes y normas. 9. Páginas web.

\section{Introducción}

Los Planes de Movilidad Urbana Sostenible (PMUS) surgen en el marco del Plan de Acción (2004-2008) de la Estrategia de Ahorro y Eficiencia Energética E4 (20042012) del Ministerio de Industria, Turismo y Comercio del Gobierno español. El órgano encargado de impulsar todo el proceso fue el Instituto de Diversificación y Ahorro Energético (IDAE). Los PMUS aparecen como un nuevo instrumento de planificación de la movilidad cuya finalidad era lograr una mayor sostenibilidad energética en los desplazamientos urbanos.

Se trataba de materializar el mandato del Programa 21 surgido de Conferencia sobre Medio Ambiente y Desarrollo celebrada en Río de Janeiro en 1992, donde ya se señalaba la necesidad de reconducir los problemas generados por el tráfico en las ciudades, y la urgencia de actuar localmente en "la planificación y la gestión del transporte urbano, en la promoción de redes de transporte urbano eficientes y ecológicamente racionales" (Ministerio de Medio Ambiente, 1998).

Un buen número de ayuntamientos españoles han hecho suyo este mandato, actuando en el ámbito local mediante la firma de la Carta de Aalborg y desarrollando Agendas Locales 21. Los Planes de Movilidad han sido una de las herramientas propuestas desde estas Agendas para atajar las afecciones sociales y ambientales. En España este proceso se ha producido únicamente en Cataluña y en el País Vasco donde a finales de los años 1990 se elaboraron algunos Planes de Movilidad en el marco de las Agendas 21(IHOBE, 1995). El resto de Planes procede del impulso dado por el IDAE con la puesta en funcionamiento del primer Plan de Acción de la E4.

Desde el comienzo de estas iniciativas son muchos los planes que se han realizado, sin embargo, existían dudas sobre la dimensión real que podían alcanzar. El objetivo de esta investigación es conocer cuál ha sido la dimensión territorial y el impacto que este instrumento de planificación de la movilidad ha tenido en nuestro país entre los años 2004 y 2014.

El presente trabajo describe por primera vez la realidad de los PMUS en España, cual ha sido su evolución a lo largo de esta década, y cuál su presencia en las distintas Comunidades Autónomas. Se señalan los distintos tipos de PMUS existentes de acuerdo al ámbito territorial.

\section{Metodología y Fuentes}

La producción científica en relación a los PMUS es pequeña al ser una herramienta de reciente implantación en España. Dentro de la disciplina geográfica encontramos 
escasas publicaciones; destaca el análisis de los PMUS de un caso concreto, la ciudad de Mérida (Gutiérrez, Jaraíz y Pérez, 2012), donde se analizan los condicionantes de la movilidad de esta ciudad: "Propuesta metodológica para diseñar modelos urbanos en colaboración: aplicación a las ciudades medias. Aplicación al caso extremeño". De estos mismos autores es también "Diseño de un modelo de asignación de viajes con aplicaciones de SIG para la gestión de Planes de Movilidad Urbana Sostenible en ciudades medias" (Gutiérrez, Jaraíz, y Pérez, 2012). Otro caso de PMUS estudiado es el del municipio de Getafe que ha ahondado en los aspectos urbanísticos el "Plan de Movilidad Urbana Sostenible (PMUS) de Getafe: una herramienta para coser la ciudad" (Vega, 2014).

Desde el ámbito institucional se ha abordado el tema de los planes de movilidad desde su puesta en funcionamiento a través de la celebración de jornadas y publicaciones. Se trata de actividades multidisciplinares con carácter formativo destinadas a las entidades locales y autonómicas. Destacan la intervención sobre "La experiencia de la participación pública en Planes de Movilidad Sostenible (Vega, 2009), el planteamiento critico de los PMUS (Santos Ganges, 2010) o el análisis del impacto social de los Planes (Vega, 2010). Ante la escasa producción científica se plantea como primer paso conocer cuál es el panorama actual de los PMUS, su dimensión e impacto sobre la población y la planificación territorial en España. La ausencia de un inventario oficial de la Administración sobre los planes existentes obliga a utilizar diversas fuentes de información que permitan una aproximación al tema. A continuación se explica el camino seguido.

Conocer el número de Planes de Movilidad Urbana Sostenible (PMUS) que existen en España ha sido una tarea difícil. En primer lugar, porque ninguna administración pública dispone de esta información para el conjunto del territorio, a pesar de que el IDAE, órgano dependiente del Ministerio de Industria, Turismo y Comercio, ha aportado el mayor volumen de subvenciones para estos Planes. Y de que, por otra parte, hayan sido las Comunidades Autónomas las que han distribuido esos presupuestos a los ayuntamientos, además de aportar, en algunos casos, otros recursos.

La tarea de confeccionar el mapa de los PMUS en España ha sido larga y complicada. Ante las lagunas informativas fue necesario un método que permitiera una aproximación suficientemente rigurosa por lo que se acudió, en primer lugar, a la fuente principal: el IDAE, la entidad que había impulsado la Estrategia de Ahorro y Eficiencia Energética - E4 y el desarrollo de los PMUS como una de las acciones específicas del sector del transporte. El IDAE facilitó los Informes Anuales de Liquidación de las Comunidades Autónomas que han permitido conocer los planes desarrollados entre 2005 y 2011. No existe información posterior porque en esa fecha el IDAE abandonó la línea de subvención de PMUS como consecuencia de los recortes económicos.

Aunque de esta forma se logró completar las primeras etapas de elaboración de PMUS, era también preciso conocer qué había pasado después de la crisis económica y de los ajustes presupuestarios del IDAE en el periodo de 2011 a 2014. Para ello se continuó investigando en dos frentes: por un lado, se realizaron búsquedas para ese 
periodo en los medios de comunicación donde con frecuencia se difunden las acciones municipales, entre ellas la decisión de elaborar o aprobar un Plan de Movilidad. Y por otro, se acudió al Ministerio de Hacienda y Administraciones Públicas, que desde 2014 otorga las subvenciones a los ayuntamientos destinadas a los servicios de transporte urbano bajo la condición de contar con un PMUS. Esto permitía incluir en el listado a todos los municipios que habían certificado la aprobación de un Plan en esa fecha.

Esta investigación ha contado con la opinión de los expertos en la materia. Para ello se realizaron entrevistas en profundidad que han permitido conocer de primera mano, el origen, el desarrollo del proceso, la problemática de su puesta en marcha, así como la valoración y el seguimiento de las acciones emprendidas.

\section{Origen y evolución}

En 1990 la Comisión Europea publicó el Libro Verde sobre medio ambiente urbano donde se señaló por primera vez la necesidad de modificar el reparto modal de la movilidad en las ciudades europeas. Se aconsejaba restringir el uso del vehículo privado y fomentar los medios ambientalmente más benignos como el transporte público y los no motorizados (Comisión Europea, 1990).

En 1994 se celebró la Conferencia Europea sobre Ciudades Sostenibles en donde se firmó la Carta de Aalborg (Dinamarca). Las ciudades firmantes se comprometían a participar en las iniciativas locales del Programa 21 de Naciones Unidas y a organizar actuaciones favorables al desarrollo sostenible, así como a iniciar la Campaña de Ciudades Europeas Sostenibles. El punto 1.9 de la Carta de Aalborg señalaba que "es imprescindible para una ciudad reducir la movilidad forzada y dejar de fomentar el uso innecesario de los vehículos motorizados. Daremos prioridad a los medios de transporte respetuosos con el medio ambiente" (Ihobe, 1995). A partir de ese momento serían muchas las ciudades europeas que pusieran en marcha Agendas Locales 21 y que centraran parte de sus esfuerzos en la reconducción de la movilidad urbana hacia la sostenibilidad.

Siguiendo los compromisos del Programa 21 de Naciones Unidas, la Unión Europea elaboró una estrategia de reconducción del modelo urbano y de transporte que dio a conocer mediante las publicaciones de la Comisión Europea; primero, con la Estrategia temática sobre medio ambiente urbano (2004) y unos años más tarde, con el Libro Verde Hacia una nueva cultura de la movilidad urbana (2009). De esta forma se estableció un nuevo modelo que reconciliaba el desarrollo económico de las ciudades y su accesibilidad, con la mejora de la calidad de vida y la protección ambiental. Reformulaba el sistema de movilidad, situando a la ciudadanía en el centro, y potenciaba los modos de transporte no motorizados y el transporte público.

Finalmente, el último Libro Blanco del Transporte: Hoja de ruta hacia un espacio único europeo de transporte: por una política de transporte competitivo y sostenible (2011) tenía por finalidad reducir las emisiones de gases de efecto invernadero en un $60 \%$ respecto a las cifras de 1990 mediante una estrategia de transporte también centrada en las áreas urbanas y suburbanas. En concreto señalaba en el punto 49 que 
"deberá animarse a las ciudades por encima de un determinado tamaño a elaborar Planes de Movilidad Urbana” (Comisión Europea, 2011).

Es cierto que diversos países europeos llevaban años trabajando en este tipo de Planes. Baste citar los primeros proyectos piloto de Plans de Désplacements Urbains (PDU) desarrollados en Francia entre 1983 y 1986 y los que posteriormente, en la década siguiente, se extendieron por las grandes aglomeraciones francesas. También surgieron en Reino Unido los denominados Local Transport Plans (LTP) o en Italia los Piani Urbani de Mobilitá (PUM) (López-Lambas y La Paix, 2008).

En España las únicas experiencias de planificación integral de la movilidad que pueden mencionarse durante los años noventa del siglo XX, antes de la aparición de las iniciativas del IDAE para implantar PMUS, fueron los proyectos asociados a los Planes de Acción de las Agendas Locales 21. Fueron los municipios de la Comunidad Autónoma del País Vasco y de Cataluña los que en esos primeros momentos afrontaron la planificación de la movilidad urbana (IHOBE, 1995). Los procesos comenzaron con medidas aisladas, destinadas a calmar el tráfico, a dulcificar la relación entre los coches y los peatones, a dar prioridad al transporte público, así como a introducir la bicicleta en la movilidad cotidiana.

Desde 1994 fueron muchos los municipios que firmaron la Carta de Aalborg y que comenzaron a implantar Agendas Locales. Estos procesos ayudaron a analizar localmente los problemas generados por la movilidad en las ciudades, y desarrollaron Planes de Acción con una visión integral del transporte que tenía en cuenta todas las movilidades.

En el caso de Cataluña, la Diputación de Barcelona creó en 1997 la Red de Ciudades y Pueblos hacia la Sostenibilidad y desarrolló el sistema municipal de indicadores sostenibles. A partir de ese contexto, el transporte centró gran parte de las problemáticas de la sostenibilidad local y las Agendas 21 convirtieron la movilidad en un aspecto esencial de los Planes de Acción (http://www.diba.cat/web/xarxasost).

El proceso en el País Vasco fue similar. En 2002 se creó la Red Vasca de Municipios hacia la Sostenibilidad (Udalsarea 21), un foro de coordinación y cooperación que dinamizó las Agendas Locales 21 de los 197 municipios vascos, y que impulsó la ejecución de los Planes de Acción. Esta red ha financiado el desarrollo de las Agendas Locales y ha permitido analizar el estado ambiental de muchas ciudades, donde el transporte era un sector clave. Los Planes de Acción han realizado propuestas sectoriales de planificación de la movilidad desde una perspectiva sostenible, y en algunos casos, dieron como resultado lo que por aquel entonces se llamaba Planes de Movilidad Urbana (http://www.udalsarea21.net).

En 2004, para facilitar el trabajo a los ayuntamientos, la Sociedad Pública de Gestión Ambiental del Gobierno Vasco publicó la Guía metodología sobre la elaboración de planes de movilidad sostenible. Este documento surgía en el contexto de la Estrategia Ambiental Vasca de Desarrollo Sostenible (2002-20) que señalaba entre sus metas alcanzar un equilibrio territorial y avanzar hacia una movilidad más sostenible. Es la primera guía que se publicó en España para orientar a los equipos técnicos y a los políticos en la elaboración de Planes de Movilidad Sostenible (IHOBE, 2004). 
En el resto de las Comunidades Autónomas también se desarrollaron procesos de implantación de Agendas Locales 21. Sin embargo, no se tiene constancia de que hayan profundizado con la necesaria intensidad en los aspectos relacionados con la movilidad, y que hayan abierto procesos de implantación de Planes de Movilidad, como lo hicieron en Cataluña y en el País Vasco.

A partir de la aprobación de la Estrategia de Ahorro y Eficiencia Energética-E4 (2004-2012) se sentaron las bases para el desarrollo de Planes de Movilidad Urbana Sostenible (PMUS) en España. La E4 desarrolló un primer Plan de Acción (2005-07) que contemplaba 31 medidas, entre las que se encontraba la Medida 13, sobre la nueva financiación de los PMUS cuyo objetivo era la reducción del consumo en 44 millones de barriles de petróleo, lo que suponía el 10\% de las importaciones. Los objetivos en esos momentos se centraban en las ciudades de más de 50.000 habitantes y el horizonte temporal era 2012. Se trataba de "actuar sobre la movilidad urbana para conseguir cambios importantes en el reparto modal, con una mayor participación de los medios más eficientes de transporte, en detrimento de la utilización del uso del vehículo privado con baja ocupación y fomentar el uso de modos no consumidores de energía fósil como la marcha a pie y la bicicleta" (Ministerio de Industria, Turismo y Comercio, 2004).

Posteriormente se puso en funcionamiento el segundo Plan de Acción (2008-12) que continuaba con las orientaciones del primero. Actualmente está en marcha un tercero (2011-20) que quería intensificar aún más el ahorro y la eficiencia energética mediante 20 medidas, la primera de las cuales se ocupaba de la financiación específica para ejecutar los planes de movilidad. El objetivo era ahorrar el $6 \%$ de las importaciones de petróleo crudo $^{2}$. Hay que señalar que estas previsiones, como luego se explicará, se han visto alteradas por la irrupción de la crisis económica.

Por otro lado, el Plan Nacional de Asignación de Derechos de Emisión, PNADE (2004-2007) que entró en vigor en septiembre de 2004 determinaba la hoja de ruta para el cumplimiento del Protocolo de Kioto. En los últimos años España incrementó las emisiones de gases de invernadero debido al crecimiento de la movilidad motorizada en los entornos urbanos, convirtiéndose en una de las actividades más contaminante $^{3}$. Era necesario que el sector del transporte se transformara rápidamente. El PNADE contemplaba los PMUS entre las medidas que debían ponerse en marcha para lograr el cambio modal (Ministerio de Industria, Turismo y Comercio, 2004).

En 2004 se redactó el Plan Estratégico de Infraestructuras y Transporte (PEIT) que buscaba adaptarse al escenario de ahorro y eficiencia energética fijado por la E4. Establecía las bases del cambio modal hacia un mayor reequilibrio entre todas las formas de transporte en el horizonte en 2020. En relación con los sistemas de transporte urbano y metropolitano definía también los Planes de Movilidad Sostenible

2 El ahorro energético es coherente con los escenarios de consumo de energía final y primaria de la Directiva 2009/28/CE de 23 de abril del 2009 y con los objetivos de reducción de gases de invernadero fijados para España en el marco de la "Estrategia 20/20/20 de la Unión Europea".

3 Las emisiones de CO2 han pasado de 58.505,8 kt de CO2 eq (1990) a 93.956 kt CO2 eq (2002). 
como instrumento de actuación (Ministerio de Fomento 2004). El posterior PITVI (2012-2024) recogía igualmente esta iniciativa (Ministerio de Fomento, 2012).

Por otro lado, la Ley 34/2007 de Calidad del Aire y Protección Atmosférica obligó a las Administraciones Públicas a adoptar prácticas adecuadas para reducir o evitar la contaminación atmosférica; y en su artículo 18 decía que era necesario integrar la protección atmosférica en las políticas sectoriales. Esta recomendación animó a muchas ciudades a implantar PMUS que integraban políticas favorables a la mejora de la calidad del aire.

La Estrategia Española de Cambio Climático y Energía Limpia - EECCEL ${ }^{4}$ (Consejo Nacional del Clima, 2007), que formaba parte de la Estrategia Española de Desarrollo Sostenible-EEDS (Ministerio de la Presidencia, 2007), dedicaba el apartado 3.3.7. a los "sectores difusos", entre los que se encuentra el transporte, para el que fijaba objetivos diferentes en diversas áreas: integrar los criterios ambientales en la planificación territorial y de las infraestructuras, lograr el cambio modal para reequilibrar el modelo actual, aumentar la eficiencia energética de los distintos modos de transporte, reducir las emisiones de gases de invernadero y otros contaminantes, así como mejorar la calidad del aire en entornos urbanos y desarrollar medidas de gestión de la demanda en ámbitos urbanos.

En 2014 el Comité de las Regiones de la Comisión Europea sacó a la luz el Paquete de Movilidad Urbana en el que se definía el "concepto de PMUS" y se generaba la estructura necesaria para "hacer realidad una movilidad urbana sostenible". Para ello la Comisión debía colaborar "en el desarrollo del concepto y de los instrumentos necesarios, mediante una plataforma europea de apoyo a los entes locales responsables de la planificación". Una de las ideas que recogía este documento de trabajo era lograr un "centro virtual de conocimiento y competencia" que diera apoyo a los entes nacionales, regionales y locales en el desarrollo y en la ejecución de los planes, incluso mediante instrumentos financieros. Actualmente esta herramienta sobre Planes de Movilidad funciona integrada en el portal de Internet ELTIS (Comisión Europea, 2014).

\section{Evolución de los PMUS en España (2005-2014)}

La investigación ha permitido registrar 250 Planes de Movilidad Urbana Sostenible (PMUS) que se han desarrollado a lo largo de prácticamente diez años. Pueden distinguirse varias etapas tal y como se refleja en la Figura 1: una primera de inicio en el año 2005; una segunda etapa de impulso entre 2006 y 2008; una tercera etapa de desaceleración y cierre del Plan de Acción entre 2009 y 2011; y una última etapa desde 2011 hasta la actualidad, marcada por el obligado cumplimiento el 1 julio del 2014, del artículo 102 de la Ley 2/2011 de Economía Sostenible.

4 Aprobado por Consejo Nacional del Clima el 25 de octubre del 2007, y por el Consejo de Ministros el 2 de noviembre del 2007. 
Figura 1. Evolución de los PMUS en España (2005-2014).

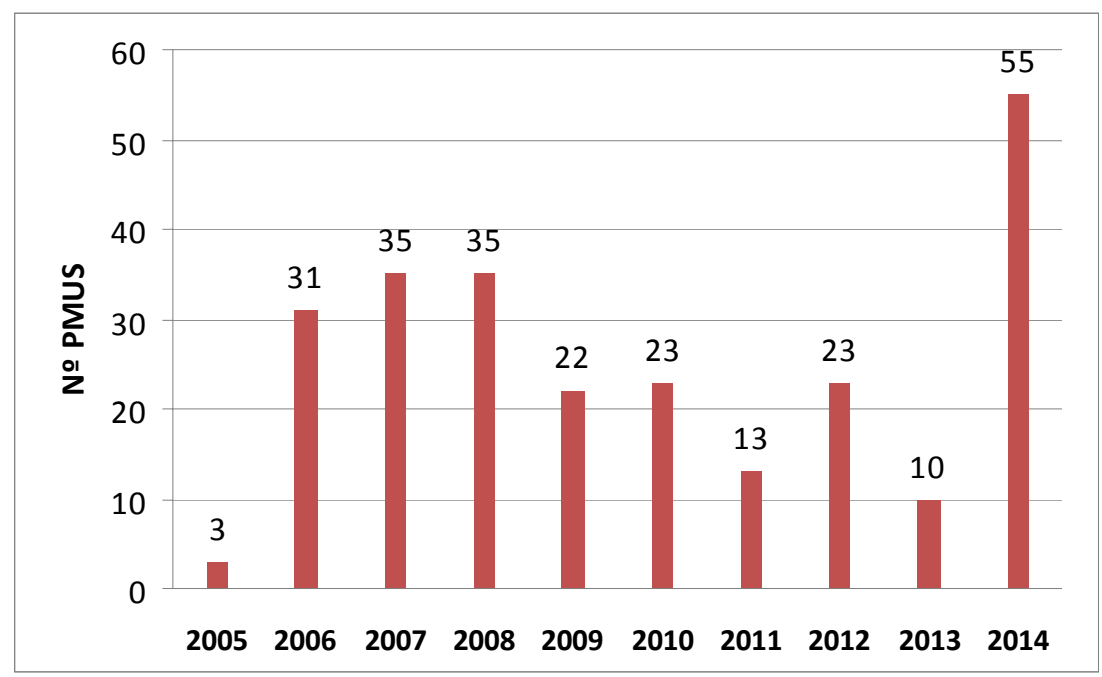

Fuente. Elaboración propia a partir 5 .

La primera etapa se corresponde con el inicio en la planificación de la movilidad sostenible y se desarrolla durante 2005. Fue el momento en que el IDAE comenzó a desarrollar incipientes experiencias en el ámbito urbano. Entre ellas se encontraba la puesta en funcionamiento de los primeros autobuses lanzadera en los polígonos industriales de Getafe. También tuvo interés por poner en marcha un Plan de Movilidad para lo que eligió el municipio de Aranjuez, una ciudad turística con un importante patrimonio; pero la redacción del Plan se realizó sin el apoyo de referencias metodológicas. Existían otras experiencias, una en San Fernando de Henares (Madrid) que participaba en el proyecto europeo Snowball y comenzó a desarrollar su Plan en 2005, y otra en Begues (Barcelona) que desarrollaba un PMU dentro de la Ley de Movilidad de Cataluña.

A partir de 2006 se inició una segunda etapa de fuerte impulso en la elaboración de PMUS que duró hasta 2008, momento en que dio comienzo la crisis económica. El IDAE buscó un municipio donde ensayar un "proyecto piloto" para la implantación de un Plan de Movilidad que permitiera poner en práctica una nueva metodología de planificación Se pretendían aplicar nuevas formas de gestionar la movilidad en municipios de tamaño y características diferentes. En aquel momento los responsables del IDAE y del Consorcio de Transportes de Madrid querían "aprender" como debía

5 Elaboración propia a partir de los Informes de Evaluación del IDAE y de las listas de subvenciones al transporte colectivo urbano del Ministerio Hacienda y Administraciones Públicas. 
realizarse un Plan de Movilidad ${ }^{6}$. En el primer cuatrimestre de 2006 se adjudicó el PMUS de Getafe y en el mes de julio se publicó la Guía práctica para la elaboración e implantación de Planes de Movilidad Urbana Sostenible (IDAE, 2006).

En este periodo el Gobierno español a través del IDAE dio un fuerte impulso al desarrollo de PMUS mediante la firma de Convenios de Colaboración con las Comunidades Autónomas para la adjudicación de subvenciones. Además, ofrecía asesoramiento técnico para apoyar las distintas acciones y proporcionar información, formación y divulgación, allí donde fuera necesario.

Con la crisis económica se inició una nueva fase, que comprende desde 2009 hasta 2011, caracterizada por un descenso en la elaboración de planes y en la que el IDAE redujo los presupuestos destinados a la financiación de PMUS hasta abandonar finalmente esta línea de trabajo en 2011. Durante este periodo se realizaron 68 planes, algunos de ellos fruto de Convenios firmados con las Comunidades Autónomas y cuya gestión se había retrasado.

La última etapa, que comenzó en 2012 y llega hasta la actualidad, está marcada por la condición impuesta por el artículo 102 de la Ley 2/2011 de 4 de marzo de Economía Sostenible, que obligaba a los ayuntamientos que desearan disponer de las ayudas al transporte público urbano, a tener aprobado un Plan de Movilidad Urbana Sostenible. La entrada en vigor definitiva de la ley se fue posponiendo hasta el 1 de julio de 2014, cuando el Ministerio de Administraciones Publicas hizo obligatorio este criterio para la asignación de las ayudas. En este periodo se han elaborado 98 planes, de los que más de la mitad lo fueron 2014, fruto de la necesidad que muchos ayuntamientos tenían para poder acogerse a esas ayudas.

\section{Los PMUS en el territorio}

\subsection{Distribución territorial en las Comunidades Autónomas}

Los 250 Planes de Movilidad señalados en el apartado anterior abarcan 405 municipios donde residen 27.307.787 habitantes. Se han diferenciado los PMUS elaborados por un único ayuntamiento, de aquellos elaborados conjuntamente con otros municipios. Algunos participan en Planes Mancomunados, Comarcales o Metropolitanos; es decir, se trata de ayuntamientos que debido a su proximidad geográfica, a que cuentan con sistemas de transporte compartidos, o a que disponen de una figura jurídica que les permite colaborar con otros para reducir esfuerzos económicos y humanos, deciden compartir esta herramienta de planificación de la movilidad; se han registrado 18 planes de este tipo. En la Figura 2, se relacionan los tipos de PMUS con el número de municipios y con la población afectada.

6 Según las declaraciones de Ángel Cediel en la entrevista realizada 26 de junio del 2013, responsable de la División de Transportes del Instituto de Desarrollo y Diversificación Energético (IDAE). 
Figura 2. Tipos de PMUS en relación al $\mathrm{N}^{\mathrm{o}}$ de municipios y a la población.

\begin{tabular}{|c|c|c|c|c|c|}
\hline & $\begin{array}{c}\text { PMUS } \\
\text { municipales }\end{array}$ & $\%$ & $\begin{array}{c}\text { PMUS } \\
\text { agrupados }\end{array}$ & $\%$ & TOTAL PMUS \\
\hline $\mathrm{N}^{\circ}$ planes & 232 & 92,8 & 18 & 7,2 & 250 \\
\hline $\mathrm{N}^{\circ}$ municipios & 217 & 53,5 & 188 & 46,4 & 405 \\
\hline $\mathrm{N}^{\circ}$ habitantes & 25.522 .703 & 93,4 & 1.785 .084 & 6,5 & 27.307 .787 \\
\hline
\end{tabular}

Fuente: Elaboración propia ${ }^{7}$.

Por Comunidades Autónomas, Andalucía es, con diferencia, la que concentra un mayor número de PMUS con 69, un 27,6\% de todo el país, siendo 153 los municipios andaluces en los que se desarrollan los planes, un 37,78\% del total. Le sigue en importancia Cataluña, con 38 Planes de Movilidad, lo que representa un 15,2\% de todos los PMUS, y el País Vasco con 32 planes, es decir un 12,8\%. Entre estas tres autonomías concentran el $65,7 \%$ de los planes de movilidad españoles. Otras autonomías, que también han sido activas en el desarrollo de planes de movilidad, son Madrid con un 9,2\%, la Comunidad Valenciana con un 6,4\%, y Castilla y León con un $5,6 \%$. Con un nivel de compromiso inferior se encontraría la Comunidad Autónoma de las Islas Baleares con un 4,8\%, y con 3,2\% Aragón, Castilla La Mancha y Cantabria, tal y como se observa en las siguientes figuras 3 y 4 .

Conviene analizar un poco más en detalle los PMUS agrupados ya que pueden dar una impresión equivocada de la dimensión real que tiene la planificación de la movilidad en algunos territorios. Se han contabilizado 188 municipios con PMUS que incluyen a varios ayuntamientos, casi la mitad de los municipios registrados $(46,41 \%)$, sin embargo tan solo representan el 6,5\% de la población que cuenta con planes de movilidad. Esta desproporción se debe a que este tipo de PMUS se desarrollan por lo general en municipios pequeños, ubicados en zonas rurales, con bajas densidades de población y una ordenación territorial dispersa (Ver figura 3 y 4).

Estos planes agrupados se caracterizan por tener un carácter más general, su menor nivel de detalle y sus propuestas genéricas. Buena parte de estos planes están localizados en áreas rurales, como el PMUS de la Mancomunidad de Municipios Sostenibles de Cantabria, pero también entornos urbanosperiurbanos como el PMUS del Área Metropolitana de Jaén o en zonas turísticas como el PMUS de la Mancomunidad de Uribe Kosta. Se trata de municipios con problemáticas similares y que tienen como objetivo principal mejorar las relaciones de transporte metropolitano o comarcal, dejando en un segundo plano las propuestas relacionadas con la problemática interna de cada municipio.

Elaboración propia a partir de los Informes de Evaluación del IDAE y de las listas de subvenciones al transporte colectivo urbano del Ministerio Hacienda y Administraciones Públicas 
Figura 3. Distribución de los PMUS por Comunidades Autónomas (2005-14).

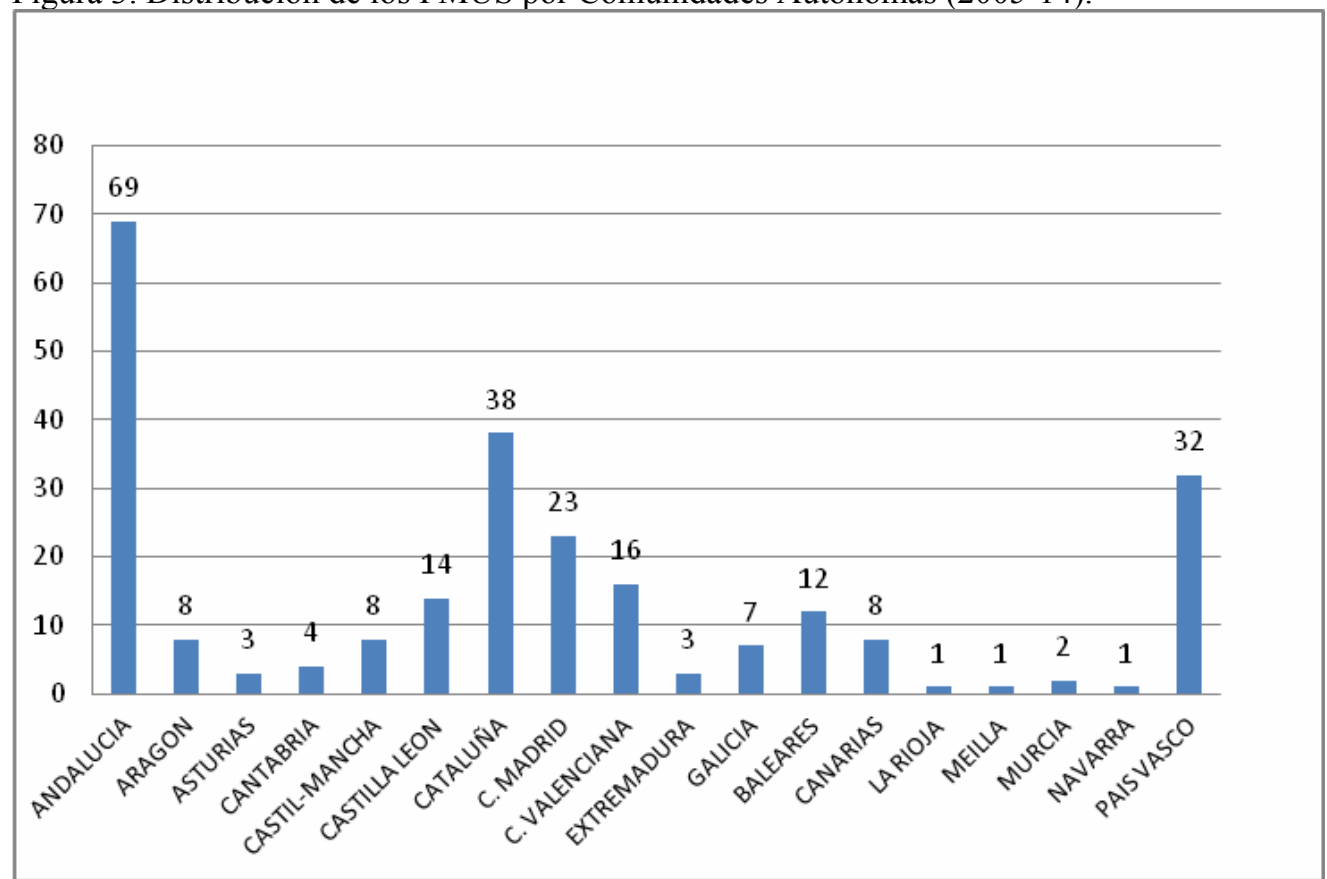

Fuente: Elaboración propia ${ }^{8}$.

Hay que señalar que Andalucía cuenta con ocho PMUS mancomunados que agrupan al $60 \%$ de los municipios con planes de movilidad, unos 92, pero representa tan solo al 10\% de la población andaluza con Planes. En Canarias hay registrados tres PMUS de este tipo que incluyen a 23 municipios, y cuya iniciativa ha partido de los Cabildos Insulares; la Comunidad Autónoma del País Vasco cuenta con dos PMUS comarcales que incluyen a 47 municipios y que se canalizan a través de Udalsarea21, el órgano del Gobierno Vasco que desarrolla la implantación de las Agendas Locales 21. En la Comunidad Foral de Navarra hay 19 municipios que se agrupan en un solo PMUS, el de la Comarca de Pamplona. Y por último, en Cantabria, la Mancomunidad de Municipios Sostenibles cuenta con un PMUS que forma parte del desarrollo del Plan de Acción de la Agenda Local 21.

8 Elaboración propia a partir de los Informes de Evaluación del IDAE y de las listas de subvenciones al transporte colectivo urbano del Ministerio Hacienda y Administraciones Públicas. 
Figura 4. Municipios con PMUS por Comunidades Autónomas, 2005-2014.

\begin{tabular}{|c|c|c|c|c|}
\hline CCAA & PMUS & $\mathbf{\%}$ & $\begin{array}{c}\mathbf{N}^{\mathbf{0}} \text { municipios } \\
\text { con PMUS }\end{array}$ & $\mathbf{\%}$ \\
\hline ANDALUCIA & 69 & 27,6 & 153 & 37,8 \\
\hline ARAGON & 8 & 3,2 & 3 & 1,98 \\
\hline ASTURIAS & 3 & 1,2 & 28 & 6,74 \\
\hline CANARIAS & 8 & 3,2 & 9 & 2,22 \\
\hline CANTABRIA & 4 & 1,6 & 38 & 9,38 \\
\hline CATALUNA & 38 & 15,2 & 14 & 1,98 \\
\hline CASTILLA LA MANCHA & 8 & 3,2 & 7 & 3,46 \\
\hline CASTILLA Y LEON & 14 & 5,6 & 3 & 1,73 \\
\hline GALICIA & 7 & 2,8 & 12 & 0,74 \\
\hline EXTREMADURA & 3 & 1,2 & 23 & 2,96 \\
\hline ISLAS BALEARES & 12 & 4,8 & 1 & 5,68 \\
\hline MADRID & 23 & 9,2 & 2 & 0,25 \\
\hline MELILLA & 1 & 0,4 & 19 & 0,49 \\
\hline MURCIA & 2 & 0,8 & 60 & 4,69 \\
\hline NAVARRA & 1 & 0,4 & 1 & 14,81 \\
\hline PAIS VASCO & 32 & 12,8 & 16 & 0,25 \\
\hline LA RIOJA & 1 & 0,4 & $\mathbf{4 0 5}$ & 3,95 \\
\hline VALENCIA & 16 & 6,4 & $\mathbf{1 0 0} \%$ & $100 \%$ \\
\hline
\end{tabular}

Fuente: Elaboración propia99.

Figura 5. PMUS “agrupados" por Comunidades Autónomas y Provincias.

\begin{tabular}{|c|c|c|c|c|}
\hline CCAA & Denominación & $\begin{array}{l}N^{0} \text { PMUS } \\
\text { agrupados }\end{array}$ & $\mathrm{N}^{0}$ municipios & $\mathbf{N}^{0}$ Habitantes \\
\hline \multirow[t]{5}{*}{ ANDALUCIA } & PMUS Mancomunados en Córdoba & 2 & 22 & 124.337 \\
\hline & PMUS del Área Metropolitana Jaén & 1 & 13 & 91.748 \\
\hline & PMUS Mancomunados en Almería & 3 & 45 & 183.088 \\
\hline & PMUS Mancomunado en Cádiz & 1 & 8 & 153.083 \\
\hline & PMUS Mancomunado en Granada & 1 & 4 & 42.745 \\
\hline Subtotal & & & 92 & 595.001 \\
\hline \multirow[t]{2}{*}{ PAIS VASCO } & PMUS comarcales en Álava & 3 & 23 & 169.330 \\
\hline & PMUS comarcales en Guipúzcoa & 2 & 24 & 97.660 \\
\hline Subtotal & & & 47 & 266.990 \\
\hline \multirow{2}{*}{$\begin{array}{c}\text { ISLAS } \\
\text { CANARIAS }\end{array}$} & PMUS mancomunado en Tenerife & 1 & 10 & 184.342 \\
\hline & PMUS mancomunado Gran Canaria & 2 & 14 & 122.273 \\
\hline Subtotal & & & 24 & 307.962 \\
\hline CANTABRIA & PMUS Mancomunado en Cantabria & 1 & 6 & 46.209 \\
\hline NAVARRA & PMUS Mancomunado en Navarra & 1 & 19 & 384.580 \\
\hline TOTAL & & 18 & 188 & 2.380 .085 \\
\hline
\end{tabular}

Fuente: Elaboración propia ${ }^{10}$.

9 Elaboración propia a partir de los Informes de Evaluación del IDAE y de las listas de subvenciones al transporte colectivo urbano del Ministerio Hacienda y Administraciones Públicas.

10 Elaboración propia a partir de los Informes de Evaluación del IDAE y de las listas de subvenciones al transporte colectivo urbano del Ministerio Hacienda y Administraciones Públicas. 
Volviendo al análisis de los PMUS en su conjunto, cabe señalar que la evolución mostrada por los datos de todo el país se repite en la mayoría de las Comunidades Autónomas. Así, Cataluña, Andalucía (Figura 6), Madrid, Islas Baleares o País Vasco tienen ritmos de elaboración similares al conjunto del país. Extremadura y Melilla no registran ningún PMUS hasta después de 2012; La Rioja y el Principado de Asturias no lo harán hasta 2014.

Figura 6. Evolución de los PMUS por Comunidades Autónomas entre 2005-14.

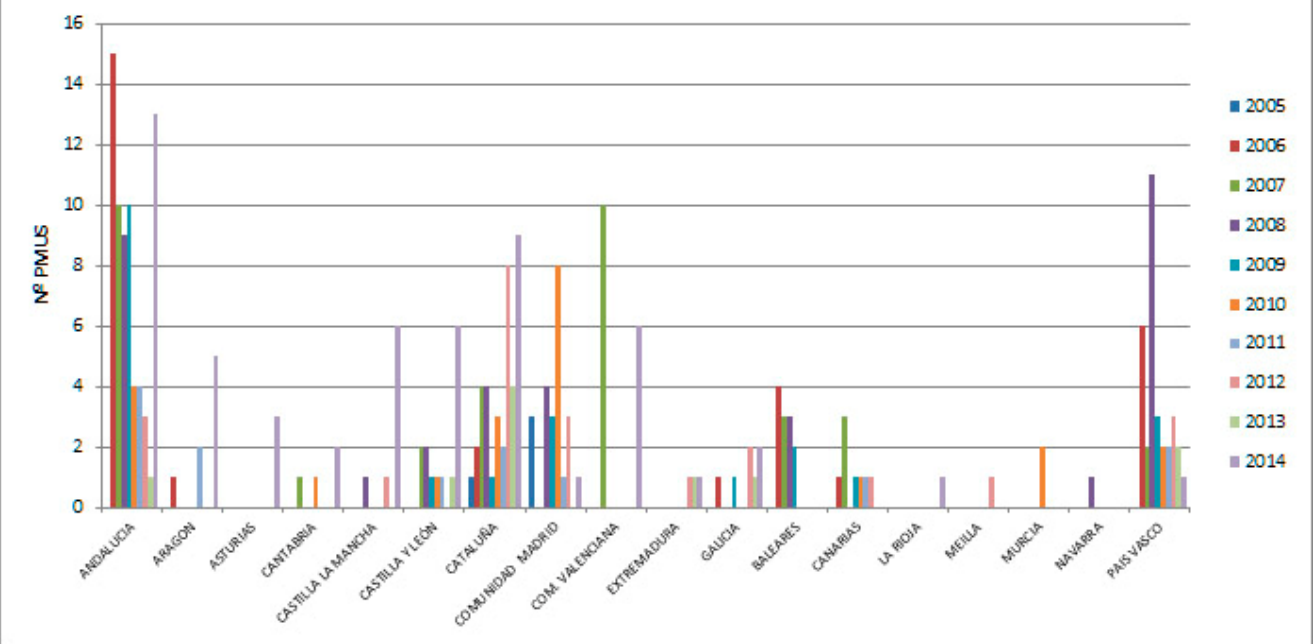

Fuente: Elaboración propia ${ }^{11}$.

En el periodo inicial del proceso fueron la Comunidad de Madrid y Cataluña las que registraron los primeros PMUS. Hay que mencionar que Cataluña partía de un proceso abierto en la elaboración de planes asociados a la Agenda 21 y que continuó su estrategia añadiendo las ayudas del IDAE. A partir de 2006 se incorporaron nuevos planes de Andalucía, del País Vasco, de Baleares y, con menos intensidad, de Galicia y de las Islas Canarias. Hasta 2008 también hicieron planes de movilidad Cantabria, Castilla y León, Comunidad Valenciana, Castilla La Mancha y la Comunidad Foral Navarra.

Hay que señalar que la mayoría de estos convenios entre el IDAE y las Comunidades Autónomas se firmaron entre 2005 y 2006, y que su tramitación hasta

11 Elaboración propia a partir de los Informes de Evaluación del IDAE y de las listas de subvenciones al transporte colectivo urbano del Ministerio Hacienda y Administraciones Públicas. 
que se asignaron las ayudas a los ayuntamientos tardó algunos años, un retraso que dependía de los distintos órganos responsables de su gestión.

El significativo incremento del número de PMUS en 2014 fue muy intenso en Andalucía, Castilla y León, Cataluña, y la Comunidad Valenciana por este orden. En algunos casos el desarrollo de los Planes coincidió con su aprobación en el ejercicio de 2014, pero en otros se aceleró para no incumplir los plazos fijados para antes del mes de julio de 2014 como sucedió con el Ayuntamiento de Madrid y con otros.

Figura 7. Evolución de los planes por Comunidades Autónomas 2005-14.

\begin{tabular}{|c|c|c|c|c|c|c|c|c|c|c|c|c|}
\hline & $\mathbf{2 0 0 5}$ & $\mathbf{2 0 0 6}$ & $\mathbf{2 0 0 7}$ & $\mathbf{2 0 0 8}$ & $\mathbf{2 0 0 9}$ & $\mathbf{2 0 1 0}$ & $\mathbf{2 0 1 1}$ & $\mathbf{2 0 1 2}$ & $\mathbf{2 0 1 3}$ & $\mathbf{2 0 1 4}$ & $\mathbf{T o t a l}$ & $\%$ \\
\hline ANDALUCIA & & 15 & 10 & & 0 & 4 & 4 & 3 & 1 & 13 & 69 & 27,6 \\
\hline ARAGON & & 1 & & & & & 2 & & & 5 & 8 & 3,2 \\
\hline ASTURIAS & & & & & & & & & & 3 & 3 & 1,2 \\
\hline CANTABRIA & & & 1 & & & 1 & & & & 2 & 4 & 1,6 \\
\hline CASTILLA MANCHA & & & & 1 & & & & 1 & & 6 & 8 & 3,2 \\
\hline CASTILLA Y LEÓN & & & 2 & 2 & 1 & 1 & 1 & & 1 & 6 & 14 & 5,6 \\
\hline CATALUÑA & 1 & 2 & 4 & 4 & 1 & 3 & 2 & 8 & 4 & 9 & 38 & 15,2 \\
\hline MADRID & 3 & & & 4 & 3 & 8 & 1 & 3 & & 1 & 23 & 9,2 \\
\hline COM. VALENCIANA & & & 10 & & & & & & & 6 & 16 & 6,4 \\
\hline EXTREMADURA & & & & & & & & 1 & 1 & 1 & 3 & 1,2 \\
\hline GALICIA & & 1 & & & 1 & & & 2 & 1 & 2 & 7 & 2,8 \\
\hline BALEARES & & 4 & 3 & 3 & 2 & & & & & & 12 & 4,8 \\
\hline CANARIAS & & 1 & 3 & & 1 & 1 & 1 & 1 & & & 8 & 3, \\
\hline LA RIOJA & & & & & & & & & & 1 & 1 & 0,4 \\
\hline MEILLA & & & & & & & & 1 & & & 1 & 0,4 \\
\hline MURCIA & & & & & & 2 & & & & & 2 & 0,8 \\
\hline NAVARRA & & & & 1 & & & & & & & 1 & 0,4 \\
\hline PAIS VASCO & & 6 & 2 & 11 & 3 & 2 & 2 & 3 & 2 & 1 & 32 & 12,8 \\
\hline TOTAL & 4 & 30 & 35 & 35 & 22 & 23 & 13 & 23 & 10 & 55 & 250 & $100 \%$ \\
\hline
\end{tabular}

Fuente: Elaboración propia ${ }^{12}$.

Como puede verse en la figuta 4 y 5 , si se tiene en cuenta el número de municipios cubiertos por los PMUS, se observa que la Comunidad de Andalucía es la que tiene un mayor número, hasta 153 municipos. Hay que señalar que esta situación se debe al importante impulso que la Agencia Andaluza de la Energía dio gracias a su colaboración con el IDAE, y a la presencia de siete planes mancomunados (Pedroches, Bajo Guadalquivir, Monachil, Bajo Andarax, Levante almeriense y Almanzora, etc) y el del Área Metropolitana de Jaén, que agrupan en total a 93

12 Elaboración propia a partir de los Informes de Evaluación del IDAE y de las listas de subvenciones al transporte colectivo urbano del Ministerio Hacienda y Administraciones Públicas. 
municipios. Como ya se ha comentado anteriormente, este tipo de planes son mas estrategicos y no permiten diseñar actuaciones detalladas.

\subsection{Impacto en el territorio y en la población de los PMUS}

Como se ha señalado anteriormente, los Planes de Movilidad han sido concebidos por las instituciones europeas como el instrumento que permite mejorar, no sólo las condiciones ambientales globales (reducción de las emisiones de gases de invernadero, disminución del consumo de combustibles fósiles, etc.), sino también la calidad ambiental de los entornos urbanos y de las condiciones de vida de quienes residen en ellos.

Por tanto, un Plan de Movilidad solo puede suponer ventajas para la ciudad afectada, tanto para los residentes como para el territorio en su conjunto. La aplicación de medidas como el calmado de tráfico, la reducción del estacionamiento de automóviles, la ampliación del espacio peatonal y ciclista, o la potenciación del transporte público, son algunas de esas propuestas con consecuencias positivas. Entre los principales beneficios se encuentran la mejora de la salud de los residentes, la reducción de los accidentes de tráfico, el incremento de los valores inmobiliarios, el aumento de las relaciones vecinales, la mejora de la calidad del aire, o la disminución del ruido.

Por este motivo es necesario conocer el volumen de población beneficiada por este instrumento de planificación de la movilidad. Igualmente es de interés dimensionar el territorio que se ve afectado por su intervención. Para ello se ha procedido en primer lugar a relacionar cada municipio con PMUS con las variables del número de habitantes, utilizando la revisión del padrón municipal en 2014 (INE, 2014).

La población beneficiada por las medidas de los PMUS en España, es decir, los habitantes cuyos municipios cuentan con un plan, alcanza a 27.307 .787 habitantes, un $58 \%$ de la población española, tal y como aparece en la figura 6.

Más de la mitad de la población que cuenta con PMUS (55,5\%) se concentra en tres Comunidades Autónomas: Madrid, con un 19,4\% de la población española (5.307.464 habitantes), Cataluña, con un 19,2\% (5.245.223 habitantes), y Andalucía que aporta un 16,9\% (4.610.945 habitantes). Otro 16,11\% de la población con PMUS se encuentra en Galicia y en la Comunidad Valenciana (Figura.8). En estas cinco comunidades se concentran las mayores ciudades y sus áreas metropolitanas y son al mismo tiempo los espacios con mayores problemas de movilidad, por lo que la presencia de estos Planes podría resolver parte de las complicaciones derivadas de los desplazamientos.

El resto de las Autonomías aportan el 28,5\% de la población; de entre ellas, las que menos son Melilla y La Rioja, que están por debajo del 1\%. Cantabria, Extremadura y la Comunidad Foral de Navarra son otras que tampoco suman mucha población. En estos casos, al tratarse de zonas con un menor grado de urbanización, la movilidad se ve afectada por conflictos puntuales en las ciudades que es donde se implantan los PMUS. 
Figura 8. Población cubierta por PMUS, 2005-2014.

\begin{tabular}{|c|c|c|c|c|c|c|}
\hline & $\begin{array}{c}\mathbf{N}^{\mathbf{0}} \\
\text { PMUS }\end{array}$ & $\begin{array}{c}\text { Municipios } \\
\text { cubiertos }\end{array}$ & $\begin{array}{c}\mathbf{N}^{\mathbf{0}} \text { hab. } \\
\text { cubiertos }\end{array}$ & $\begin{array}{c}\mathbf{N}^{\mathbf{0}} \text { Hab. } \\
\text { CCAA }\end{array}$ & $\begin{array}{c}\text { \% sobre la } \\
\text { Autonomía }\end{array}$ & $\begin{array}{c}\text { \% sobre } \\
\text { España }\end{array}$ \\
\hline Andalucía & 69 & 153 & 4.610 .945 & 8.402 .305 & 54,88 & 16,89 \\
\hline Aragón & 8 & 8 & 833.488 & 1.349 .467 & 61,76 & 3,05 \\
\hline Asturias & 3 & 3 & 540.513 & 1.061 .756 & 50,91 & 1,98 \\
\hline Islas Canarias & 8 & 28 & 1.246 .041 & 2.105 .000 & 59,19 & 4,56 \\
\hline Cantabria & 4 & 9 & 308.285 & 588.656 & 52,37 & 1,13 \\
\hline Cataluña & 38 & 38 & 5.245 .223 & 7.518 .903 & 69,76 & 19,21 \\
\hline Castilla La Mancha & 8 & 8 & 584.265 & 2.078 .611 & 28,11 & 2,14 \\
\hline Castilla y León & 14 & 14 & 1.212 .407 & 2.494 .790 & 48,60 & 4,44 \\
\hline Galicia & 7 & 7 & 1.904 .806 & 2.748 .695 & 69,30 & 6,98 \\
\hline Extremadura & 3 & 3 & 305.357 & 1.099 .632 & 27,77 & 1,12 \\
\hline Islas Baleares & 12 & 12 & 662.020 & 1.332 .019 & 49,70 & 2,42 \\
\hline Madrid & 23 & 23 & 5.307 .464 & 6.454 .440 & 82,23 & 19,44 \\
\hline Melilla & 1 & 1 & 84.509 & 84.509 & 100,00 & 0,31 \\
\hline Murcia & 2 & 2 & 690.293 & 1.466 .818 & 47,06 & 2,53 \\
\hline Navarra & 1 & 19 & 348.580 & 640.790 & 54,40 & 1,28 \\
\hline País Vasco & 32 & 60 & 1.052 .870 & 2.188 .985 & 48,10 & 3,86 \\
\hline La Rioja & 1 & 1 & 151.962 & 319.002 & 47,64 & 0,56 \\
\hline Valencia & 16 & 16 & 2.218 .759 & 5.004 .844 & 44,33 & 8,13 \\
\hline TOTAL & $\mathbf{2 5 0}$ & $\mathbf{4 0 5}$ & $\mathbf{2 7 . 3 0 7 . 7 8 7}$ & $\mathbf{4 6 . 9 3 9 . 2 2 2}$ & $\mathbf{5 8 , 1 8}$ & $100 \%$ \\
\hline
\end{tabular}

Fuente: Elaboración propia ${ }^{13}$.

El otro indicador de interés es el territorio afectado por Planes de Movilidad, es decir, la superficie donde se aplican políticas favorables a la movilidad sostenible. Se trata de espacios donde se han implantado o podrán hacerlo en un futuro medidas que contribuyan a la transformación de ese territorio. De tal forma que las intervenciones propuestas supondrían siempre una mejora de la situación de partida con reducción de los impactos, adecuada integración de los sistemas de transporte o disminución de la contaminación atmosférica y del ruido, entre otras muchas ventajas posibles.

Las actuaciones se materializarían en el rediseño de sus infraestructuras que contribuirían a una menor fragmentación de los barrios y las vecindades, una mayor permeabilidad de sus calles y su espacio público o la renaturalización de los entornos urbanos. Algunas de las intervenciones estarían relacionadas con una distribución equilibrada de la ocupación de espacio por las infraestructuras de los distintos modos de transporte; ello traería consigo un mayor protagonismo de las redes peatonales, ciclistas y de la red de transporte colectivo, la reconversión de las áreas de

13 Elaboración propia a partir de los Informes de Evaluación del IDAE y de las listas de subvenciones al transporte colectivo urbano del Ministerio Hacienda y Administraciones Públicas. 
estacionamiento para otros usos, o la transformación de autopistas en corredores verdes.

La superficie cubierta por Planes de Movilidad es una parte muy pequeña del territorio: un 9,8\%, que se corresponde con 49.840,62 km2 de la superficie de España. La figura 9 ilustra el territorio por los municipios que cuentan con PMUS en la actualidad.

Figura 9. Mapa de la distribución territorial de los PMUS, 2004-2014.

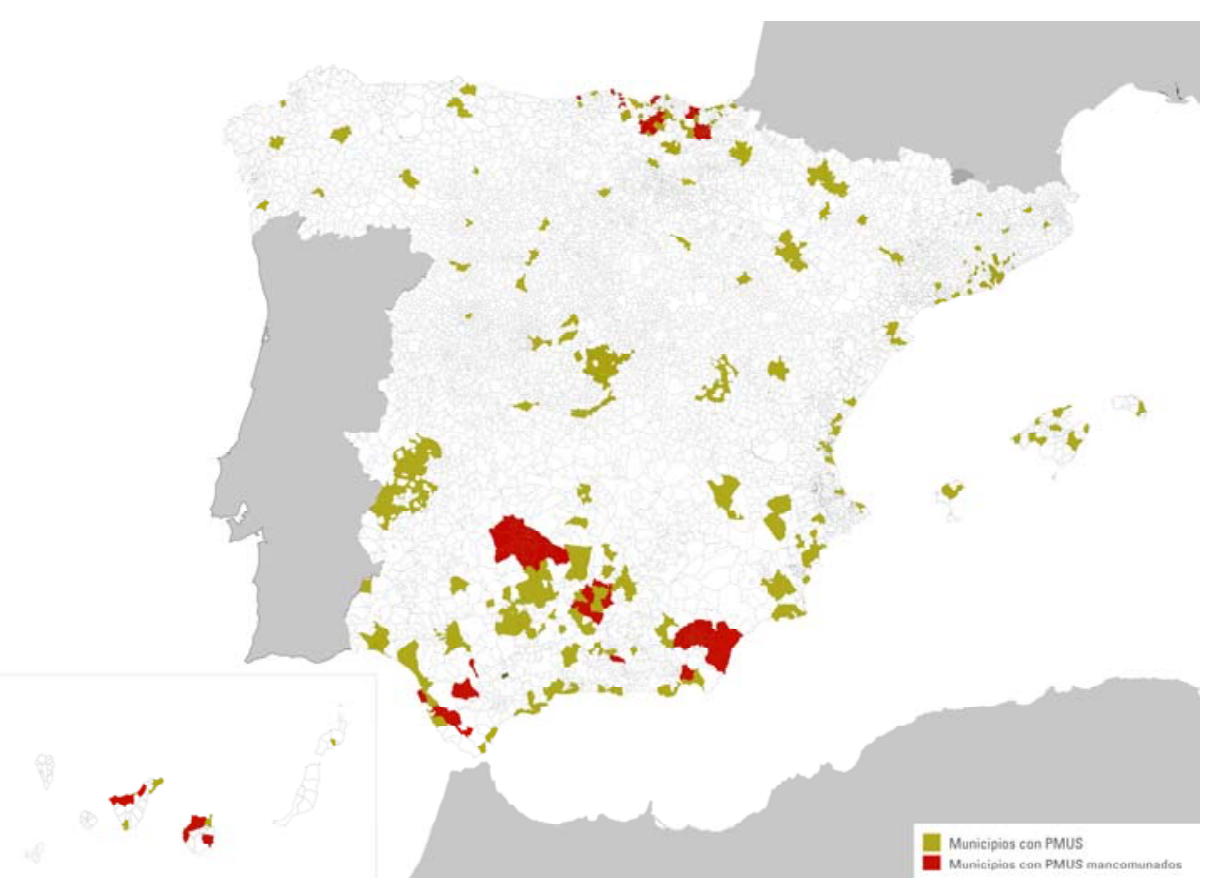

Fuente: Elaboración propia ${ }^{14}$.

Este indicador es menos ilustrativo que el referido a la población beneficiada, debido a que algunos espacios urbanizados se caracterizan por concentrar a sus habitantes en una superficie pequeña. Así, una buena parte de las ciudades con PMUS cuentan con mayores densidades de población, como es el caso de Melilla que ocupa el $100 \%$ de su superficie y cuenta con $6.870 \mathrm{hab} / \mathrm{km}^{2}$, o el País Vasco donde un

14 Elaboración propia a partir de los Informes de Evaluación del IDAE y de las listas de subvenciones al transporte colectivo urbano del Ministerio Hacienda y Administraciones Públicas. 
millón de habitantes con PMUS ocupan la mitad $(48,6 \%)$ del territorio. Canarias, Baleares y Andalucía se encuentran entre el 22 y $26 \%$ de su superficie con Planes.

Figura 10. Superficie cubierta por PMUS por Comunidades Autónomas, 2014.

\begin{tabular}{|c|c|c|c|c|c|c|}
\hline & $\begin{array}{c}\mathbf{N}^{\circ} \\
\text { PMUS }\end{array}$ & $\begin{array}{l}\text { Municipios } \\
\text { cubiertos }\end{array}$ & $\begin{array}{l}\text { Superficie cubierta } \\
\text { por PMUS Km² }\end{array}$ & Superficie CCAA & $\begin{array}{c}\text { \% sobre el total de la } \\
\text { Autonomía }\end{array}$ & $\begin{array}{c}\text { \% Superficie } \\
\text { cubierta sobre } \\
\text { superf. España }\end{array}$ \\
\hline Andalucía & 69 & 153 & $20.663,67$ & 87.597 & 23,59 & 4,09 \\
\hline Aragón & 8 & 8 & $2.848,00$ & 47.719 & 5,97 & 0,56 \\
\hline Asturias & 3 & 3 & 514,38 & 10.604 & 4,85 & 0,10 \\
\hline Islas Canarias & 8 & 28 & $1.665,00$ & 7.493 & 22,22 & 0,33 \\
\hline Cantabria & 4 & 9 & 96,77 & 5.321 & 1,82 & 0,02 \\
\hline Cataluña & 38 & 38 & $2.689,00$ & 32.108 & 8,37 & 0,53 \\
\hline Castilla Mancha & 8 & 8 & $3.518,00$ & 79.463 & 4,43 & 0,70 \\
\hline Castilla y León & 14 & 14 & $2.082,60$ & 94.226 & 2,21 & 0,41 \\
\hline Galicia & 7 & 7 & 673,00 & $29.574,3$ & 2,28 & 0,13 \\
\hline Extremadura & 3 & 3 & $4.056,00$ & 41.635 & 9,74 & 0,80 \\
\hline Islas Baleares & 12 & 12 & $1.302,00$ & 4.992 & 26,08 & 0,26 \\
\hline Madrid & 23 & 23 & $1.860,45$ & 8.030 & 23,17 & 0,37 \\
\hline Melilla & 1 & 1 & 12,30 & 12,30 & 100,00 & 0,002 \\
\hline Murcia & 2 & 2 & $2.049,72$ & 11.313 & 18,12 & 0,41 \\
\hline Navarra & 1 & 19 & 398,10 & 10.391 & 3,83 & 0,08 \\
\hline País Vasco & 32 & 60 & $3.516,08$ & 7.234 & 48,60 & 0,70 \\
\hline La Rioja & 1 & 1 & 79,57 & 5.045 & 1,58 & 0,02 \\
\hline Valencia & 16 & 16 & $1.815,98$ & 23.255 & 7,81 & 0,36 \\
\hline TOTAL & 250 & 405 & $49.840,62$ & 504.645 & & 9,88 \\
\hline
\end{tabular}

Fuente: Elaboración propia $^{15}$.

Por Comunidades Autónomas, los municipios con PMUS de Andalucía son los que más extensión ocupan, llegando a $20.663,67 \mathrm{~km}^{2}$, un $4 \%$ de total de España (lo que representa un $23,5 \%$ de la superficie andaluza). Hay otras autonomías como Extremadura que, a pesar de contar tan sólo con tres Planes de Movilidad, ocupan 4.056 Km2, un 0,8\% de la superficie de España; o Castilla La Mancha, con 3.518 $\mathrm{km} 2$. Por el contrario, otros territorios con muchos planes de movilidad tienen poco impacto territorial, como es el caso del País Vasco, con tan solo $3.516 \mathrm{~km}^{2}$; y Cataluña, con $2.689 \mathrm{~km}^{2}$.

Esto significaría que, en este momento o en un futuro no muy lejano, el señalado por los Planes de Actuación de los PMUS, los residentes en estas zonas podrán disfrutar de los beneficios de las medidas programadas. Esta afirmación requiere

15 Elaboración propia a partir de los Informes de Evaluación del IDAE y de las listas de subvenciones al transporte colectivo urbano del Ministerio Hacienda y Administraciones Públicas. 
muchos matices ya que para implantar los PMUS es imprescindible su puesta en marcha y el impulso político necesario para que sus objetivos se conviertan en realidad.

\section{Conclusiones}

La planificación de la movilidad urbana sostenible en España ha contado con una estructura en cascada, coordinada y armada a partir de estrategias territoriales, energéticas y del transporte en todos los ámbitos territoriales, desde el autonómico al local. En este sentido, la puesta en marcha de la Estrategia de Ahorro y Eficiencia Energética, E4 (2004-2012) se materializó en el ámbito local mediante los PMUS, un instrumento adecuado para reducir el consumo energético generado por la movilidad urbana. Aunque las primeras experiencias surgieron en el marco de los Planes de Acción de las Agendas Locales 21, no tuvieron la trascendencia necesaria para implicar a todas las Comunidades Autónomas, y únicamente se desarrollaron planes de ese tipo en el País Vasco y en Cataluña.

En este proceso, el IDAE ha sido una pieza fundamental, no sólo porque asignaba ayudas presupuestarias, sino también por su colaboración en tareas de asesoramiento, información, formación y divulgación de los PMUS. Sin embargo, la ausencia de inventario, seguimiento y evaluación de los PMUS que permitiera conocer el cumplimiento de los programas y las medidas iniciales, hizo que la labor de esta entidad quedase ensombrecida. La Administración ha hecho dejación de sus funciones al no comprobar la aplicación de las medidas para las que había dado apoyo económico.

La investigación ha permitido constatar, entre 2005 y 2014, 250 PMUS han planificado la movilidad de 405 municipios en los que residen 27,3 millones de habitantes. Estos planes tiene programadas actuaciones en casi $50.000 \mathrm{~km}^{2}$, aproximadamente el $10 \%$ de la superficie del país, que, aunque pudiera parecer un porcentaje pequeño del territorio, corresponde en su mayoría a zonas urbanas con alta densidad de población. Hay también otros planes elaborados por mancomunidades o comarcas que afectan a 188 municipios de menor entidad, localizados en zonas rurales o periurbanas con densidades más bajas.

En cuanto a la distribución territorial, tres Comunidades Autónomas (Andalucía, Cataluña y País Vasco) concentran el $65,5 \%$ de todos los PMUS. Con una participación inferior se encuentran la Comunidad de Madrid, la Comunidad Valenciana, Castilla y León y las Islas Baleares que tienen el 25,4\% de los planes, mientras el resto de comunidades registran porcentajes inferiores. Algunas autonomías, donde diversos municipios se agrupan para redactar el Plan, concentran un buen número de PMUS. Esta figura mancomunada podría ser de enorme utilidad para ejecutar actuaciones intermunicipales relacionadas con la operatividad del transporte público, la configuración de calmado de tráfico en carreteras interurbanas o para la gestión de sistemas de coche compartido. Sin embargo, en estos casos, el grado de definición de las medidas es muy genérico lo que impide concretar las posibles actuaciones. 
La crisis económica, que supuso la desaparición de las subvenciones del IDAE a partir de 2011, ha condicionado el desarrollo de los Planes de Movilidad porque ha reducido el ritmo de estas iniciativas o las ha paralizado. Ha sido necesaria instrumento legal para continuar la elaboración e implantación de PMUS: la entrada en vigor de la Ley 2/2011 de Economía Sostenible. El desarrollo normativo de la citada ley establecía la obligación de que los ayuntamientos dispusieran de un PMUS para acceder a las subvenciones del transporte colectivo urbano a partir del 1 de julio 2014 (Ministerio de Hacienda y Administraciones Públicas, 2014). Después de una etapa de estancamiento, la norma hizo que se aprobaran en ese año el $22 \%$ de los Planes. Este nuevo marco regulador delegó en las corporaciones locales, no sólo la decisión de realizar o renovar los PMUS, sino también el compromiso presupuestario para su desarrollo y ejecución. Pero esta medida continúa sin incluir mecanismos de control: no se revisan los contenidos de los Planes, no se valora la sostenibilidad de las medidas que incluyen, ni tampoco su cumplimiento.

Sigue siendo necesario un inventario oficial por parte de la Administración Pública que permitiera, por un lado dimensionar la presencia de estos planes y, por otro, evaluar de forma rigurosa los indicadores de control para lograr los objetivos de ahorro y eficiencia energética. De esta forma, las ayudas que otorga el estado se corresponderían con los criterios de sostenibilidad.

\section{Referencias bibliográficas}

Comisión de las Comunidades Europeas. (1990): Libro Verde sobre el medio ambiente urbano. Bruselas: Oficina de Publicaciones Oficiales de la Comisión Europea. Bruselas.

Comision Europea (2001): Libro Blanco del Transporte, La política Europea de transportes de cara al 2010: La hora de la verdad.

Comision Europea (2011): Libro Blanco del Transporte: Hoja de ruta hacia un espacio único europeo de transporte: por una política de transportes competitiva y sostenible.

Comisión de las Comunidades Europeas. (2007): Libro Verde del Transporte urbano: Hacia una nueva cultura de la movilidad. Bruselas, Oficina de Publicaciones Oficiales de la Comisión Europea.

Comisión Europea (2014): Paquete de movilidad urbana. Comisión de Política de Cohesión Territorial 19 febrero 2014.

Comisión del Medio Ambiente y del Desarrollo de Naciones Unidas (1988): Nuestro futuro común. Madrid, Alianza Editorial.

Conferencia de las Naciones Unidas sobre Medio Ambiente y Desarrollo. (1998): Programa 21. Dirección General de Calidad y Evaluación Ambiental del Ministerio de Medio Ambiente.

Conferencia EUropea sobre Ciudades Sostenibles. (1994): Carta de las Ciudades Europeas hacia la sostenibilidad. Conferencia celebrada en Aalborg (Dinamarca) el 27 de mayo de 1994.

Consejo Nacional del Clima. (2007): Estrategia Española de Cambio Climático y Energía Limpia. Horizonte 2020. Consejo Nacional del Clima de 25 de octubre del 2007, aprobado por el Consejo de Ministros el 2 de noviembre del 2007.

Diputación de Barcelona (2014). http://www.diba.cat/web/xarxasost. 
Federación Española de Municipios y Provincias (2010): Estrategia Española de Movilidad Sostenible y los Gobiernos Locales. FEMP, Red Española de Ciudades por el Clima.

Gutierrez, J.A., Jaraiz, F.J. y Perez, J.M. (2011). "Los Planes de Movilidad Sostenible: el ejemplo de la ciudad de Mérida". Universidad de Extremadura.

Gutierrez, J.A., Jaraiz, F.J. y Perez, J.M. (2012). "Diseño de un modelo de asignación de viajes con aplicación de SIG para la gestión de Planes de Movilidad Urbana Sostenible en ciudades medias". Geofocus.

International Council for Environmental Initiatives, ICLEI (1995): European Local Agenda21. Planning Guide

Instituto para la Diversificación y Ahorro de la Energía (IDAE).Ministerio de Industria, Turismo y Comercio. (2006): Guía práctica para la elaboración e implantación de Planes de Movilidad Urbana Sostenible.

López LAmbas, M.E. y La Paix, L. (2008): "Los Planes de Movilidad Urbana Sostenible en Europa". Transyt.

Ministerio de Hacienda y Administraciones Públicas (2014). Resolución de la Secretaria General de Coordinación Autonómica y Local por la que se establece, en relación a la subvención al transporte colectivo urbano interior, la forma de presentación de la información en el apartado seis del artículo 117 de la Ley de Presupuestos Generales del Estado para el 2014. Secretaria de Estado de Administraciones Públicas. Secretaria General de Coordinación Autonómica y Local.

Ministerio Industria, Turismo y Comercio (2008): Estrategia de Ahorro y Eficiencia Energética en España, E4

Ministerio de Fomento y Ministerio de Medio Ambiente, Medio Rural y Marino. (2009): Estrategia española de Movilidad Sostenible.

Ministerio de Fomento (2004): Plan Estratégico de Infraestructuras y Transporte PEIT

Ministerio de Fomento (2013): Plan de Infraestructuras, Transporte y Vivienda: PITVI (20122024). Secretaria de Estado de Infraestructuras, Transporte y Vivienda

Ministerio de Medio Ambiente (2006): Estrategia del medio ambiente urbano.

Ministerio de Medio Ambiente (2007): Estrategia Española de Cambio Climático y Energía Limpia (EECCEL). Horizonte 2007-2012-2020.

Ministerio de Presidencia (2007): Estrategia española de desarrollo sostenible.

Monzon de Caceres, A. y Lopez Lambas, E. (2009). "Planes de Movilidad Urbana ¿agentes de cambio o cambio de agentes?". Revista IT, No 86.

Santos y Ganges, L (2010). Los PMUS en España, competencias, tipos e implementación. Taller de Conferencias Sumobis. Ayuntamiento de Ponferrada. https://www.youtube.com/watch?v=947Tp3p-mPc

Sociedad Pública de Gestion Ambiental-IHOBE. (2004): Guía Práctica para la elaboración de Planes Municipales de Movilidad sostenible. Agenda Local 21. Gobierno Vasco.

Sociedad Pública de Gestión Ambiental-IHOBE (2014). http://www.udalsarea21.net . Gobierno Vasco.

Vega Pindado, P. (2007). Experiencia de participación pública en la elaboración de Planes de Movilidad Urbana Sostenible en España. Espacios Urbanos, Espacios Humanos, Hacia una movilidad Sostenible. IDAE y Consorcio de Transportes de Madrid.

Vega Pindado, P (2010). Impacto Social de los PMUS en España, competencias, tipos e implementación. Taller de Conferencias Sumobis. Ayuntamiento de Ponferrada. https://www.youtube.com/watch?v=JIosMiuo 75M 
Vega Pindado, P (2014). "El Plan de Movilidad Urbanos Sostenible de Getafe (PMUS): una herramienta para coser la ciudad". XII Coloquio y trabajos de campo del Grupo de Geografía Urbana de al AGE.

\section{Leyes y normas}

Ley 34/2007 de Calidad del Aire y Protección Atmosférica

Ley $2 / 2011$ de Economía Sostenible de 4 marzo y

Ley 2/2012 de 29 junio- disposición final 31 con efectos desde el 1 julio 2012.

Ley 9/2003 de Movilidad de Cataluña de 13 de junio

\section{Páginas web}

www.geo-focus.org

http://www.eltis.org 